\title{
MOBILIDADE DE ÍONS EM SOLO SOB SISTEMA DE SEMEADURA DIRETA SUBMETIDO ÀS ADUBAÇÕES MINERAL E ORGÂNICA ${ }^{(1)}$
}

\author{
Oromar João Bertol ${ }^{(2)}$, Maria do Carmo Lana ${ }^{(3)}, \operatorname{Emerson~Fey~}^{(4)} \&$ \\ Nivaldo Eduardo Rizzi ${ }^{(5)}$
}

\begin{abstract}
RESUMO
A retenção, no perfil do solo, de elementos minerais aplicados em sua superfície é fundamental para manter a qualidade da água e diminuir prejuízos econômicos e ambientais. Este trabalho avaliou a mobilidade do $\mathrm{K}, \mathrm{Ca}, \mathrm{Mg}$, teores de $\mathrm{Al}$ e matéria orgânica, as alterações de pH, em Latossolo Vermelho eutroférrico cultivado sob sistema de semeadura direta, bem como a demanda química de oxigênio (DQO) da água de percolação. A mobilidade foi medida em colunas de solo com estrutura preservada e submetidas a adubação mineral $\left(100 \mathrm{~kg} \mathrm{ha}^{-1}\right)$ ou orgânica $\left(150 \mathrm{~m}^{3} \mathrm{ha}^{-1}\right)$. Com uso de adubo mineral houve maior lixiviação de K, Ca e Mg, mesmo com maior quantidade de K aplicado via adubo orgânico do que via adubo mineral. A DQO da água percolada no sistema com adubação orgânica foi menor do que com adubação mineral. Os teores de $\mathrm{Ca}^{2+}$ e de $\mathrm{Mg}^{2+}$ foram maiores na camada de $\mathbf{0}$ a $2,5 \mathrm{~cm}$, principalmente para a adubação orgânica. $O$ adubo orgânico não aumentou o teor de matéria orgânica no solo, porém, na camada de 0 a $2,5 \mathrm{~cm}$, elevou o pH do solo em uma unidade e neutralizou o $\mathrm{Al}^{3+}$. Os resultados deste trabalho mostram o efeito depurador do solo sobre águas contaminadas, evidenciando os benefícios econômicos e ambientais que poderão advir com a infiltração no solo da água de enxurrada que venha a se formar em lavouras.
\end{abstract}

Termos de indexação: erosão hídrica, lixiviação, contaminação ambiental, nutrientes, dejeto líquido de suíno.

\footnotetext{
(1) Parte da Tese de Doutorado do primeiro autor, apresentada ao Setor de Ciências agrárias, Universidade Federal do Paraná UFPR. Recebido para publicação em 25 de novembro de 2009 e aprovado em 14 de abril de 2011.

(2) Eng. Agrônomo, Dr. Áreas de Meio Ambiente e geoprocessamento, EMATER. Rua da Bandeira 500, CEP 80035-270 Curitiba (PR). E-mail: oromar@emater.pr.gov.br

(3) Professora do Centro de Ciências Agrícolas, UNIOESTE. Brasil. E-mail: mclana@unioeste.br

(4) Professor do Centro de Ciências Agrícolas, UNIOESTE. Brasil. E-mail: efey@unioeste.br

(5) Professor do Curso de Engenharia Florestal, UFPR. E-mail: niva@ufpr.br
} 


\title{
SUMMARY: ION MOBILITY IN A NO-TILLAGE SOIL SUBJECTED TO MINERAL AND ORGANIC FERTILIZATION
}

\begin{abstract}
The retention in soil profiles of surface-applied mineral elements is crucial to maintain the water quality and reduce environmental and economic damages. This study evaluated: the mobility of potassium, calcium and magnesium, aluminum and organic matter contents and changes in $p H$ in an Eutroferric Red Latosol under no-tillage, and the chemical oxygen demand (COD) of percolated water. Mobility was assessed in an undisturbed soil column with preserved structure and treated with mineral $\left(100 \mathrm{~kg} \mathrm{ha}^{-1}\right)$ and organic $\left(150 \mathrm{~m}^{3} \mathrm{ha}^{-1}\right)$ fertilization. Mineral fertilization increased $\mathrm{K}, \mathrm{Ca}^{2+}$ and $\mathrm{Mg}^{2+}$ leaching, although the quantity of Kapplied was greater in the organic than in the mineral fertilizer. The COD of percolated water under organic fertilizer was significantly reduced. Soil Ca and $\mathrm{Mg}$ contents were highest in the 0$2.5 \mathrm{~cm}$ layer, mainly under organic fertilization. Organic fertilizer did not raise the soil organic matter content, but reduced soil pH by one unit and neutralized $A l^{3+}$. Results of this study show how soil plays the role of a filter of contaminated water, evidencing the economic and environmental benefits in the case of runoff infiltration from croplands.
\end{abstract}

Index terms: water erosion; leaching; environmental contamination; nutrients; liquid swine manure.

\section{INTRODUÇÃO}

O uso de dejeto de animais na agricultura representa uma fonte de nutrientes para o desenvolvimento das plantas. Em razão disso, sua aplicação nas lavouras tem se elevado nos últimos anos, particularmente no Sul do Brasil. No entanto, os dejetos têm sido distribuídos quase sempre na superfície do solo, com consequente acúmulo de elementos químicos nas primeiras camadas do solo e contaminação dos recursos hídricos, caso alcancem os mananciais de água, transportados por enxurrada que venha a se formar nas lavouras (Piovesan et al., 2009; Bertol et al., 2010).

Solos altamente intemperizados, como os Latossolos, apresentam mineralogia com marcante presença de caulinita, que participa ativamente na retenção de cátions (CTC), em razão da presença de cargas negativas de superfície (Fontes et al., 2001). A matéria orgânica, igualmente, participa da retenção de nutrientes no solo em razão de possuir CTC geralmente muito superior à dos argilominerais, proporcionada pela sua natureza coloidal e pela presença de grupos funcionais carboxílicos e fenólicos (Daniel et al., 1998; Souza et al., 2006). Portanto, a natureza mineralógica dos Latossolos, associada às propriedades coloidais da matéria orgânica, estabelece nesse solo um complexo de cargas negativas, proporcionando elevada CTC. A alta reatividade de elementos químicos, como o $\mathrm{Ca}$ e o $\mathrm{Mg}$, com os constituintes minerais e orgânicos do solo também contribui para retenção desses elementos na matriz do solo (Gomes et al., 2001; Parham et al., 2002; Conte et al., 2003); essa reatividade acentua-se com o aumento do teor de argila (Sims et al., 1998). Além disso, solos com elevado intemperismo são geralmente profundos, o que igualmente contribui para a retenção de íons (Fontes et al., 2001). Essas características, aliadas a um alto grau de deficiência de nutrientes nas camadas mais profundas (Simard et al., 2000), dão a esses solos grande capacidade de depurar águas de enxurrada que venham a percolar.

Os adubos apresentam comportamentos distintos quanto à solubilização de seus constituintes, o que influencia diretamente o processo de transporte de elementos químicos para o sistema aquático. Adubos minerais, com elevada solubilidade em água, favorecem as perdas de nutrientes solúveis em curto prazo (McDowell \& Sharpley, 2001), ao passo que adubos orgânicos, por dependerem da mineralização da fração orgânica, tornam-se uma fonte de nutrientes de longo prazo (Shigaki et al., 2006; Peles, 2007; Berenguer et al., 2008; Takalson \& Leytem, 2009).

Reduzir a erosão hídrica, principal agente da poluição difusa, e favorecer a infiltração da água no solo são medidas importantes para a melhoria da qualidade das águas e para o controle das perdas de elementos minerais das lavouras. Em face disso, é conveniente que a água da enxurrada se infiltre no solo, de modo a utilizar o potencial da matriz do solo em reter a carga iônica que lixivia com água de percolação (Prevedello, 1996). Num contexto ambiental, o solo pode exercer as funções de receber, reter e liberar elementos minerais (Larson \& Pierce 1991), bem como depurar a água de enxurrada, uma vez que tem a capacidade de reter a quase totalidade de grande parte dos elementos minerais em solução na água (Bertol et al., 2007).

Entre as várias estratégias para melhorar a qualidade da água, sistemas de terraceamento têm se apresentado como uma alternativa tecnicamente 
viável. Os terraços diminuem a velocidade do escoamento superficial e permitem que a água acumule no canal, aumentando sua infiltração (Bertoni \& Lombardi Neto, 1990; Griebeler et al., 2005; Bertol, et al., 2007), possibilitando assim a retenção dos poluentes no perfil do solo, em vez de serem lançados diretamente nas águas superficiais.

O objetivo deste trabalho foi avaliar o efeito depurador do solo sobre a água de enxurrada contaminada por adubações mineral e orgânica, percolada através de colunas indeformadas de solo cultivado sob o sistema de semeadura direta. Com essas informações, espera-se contribuir na fundamentação para adoção de práticas que favoreçam a infiltração de água no solo, entre elas o terraceamento sob semeadura direta, e, consequentemente, na redução da poluição difusa ocasionada pela erosão hídrica.

\section{MATERIAL E MÉTODOS}

Colunas indeformadas de solo, com $20 \mathrm{~cm}$ de altura e $20,3 \mathrm{~cm}$ de diâmetro, foram coletadas em novembro de 2003, no município de Marechal Cândido Rondon-PR (coordenadas UTM 194.290 e 7.283.460, fuso -22), em lavoura manejada sob sistema de semeadura direta e com terraços implantados em nível. A coleta foi realizada em uma faixa de terra entre dois terraços (área experimental), situada no terço médio de uma encosta. Segundo a classificação de Köppen, o clima da região é do tipo $\mathrm{Cfa}$, subtropical úmido, sem estação seca (Brasil, 1982). O solo da área experimental (Quadro 1) é um Latossolo Vermelho eutroférrico típico (Rhodic Hapludox), textura muito argilosa, com os seguintes atributos físicos na camada de 0 a $20 \mathrm{~cm}$ : densidade do solo $=1,29 \mathrm{~kg} \mathrm{dm}^{-3} ;$ densidade de partículas $=2,74 \mathrm{~kg} \mathrm{dm}^{-3} ;$ microporos $=0,41 \mathrm{dm}^{3} \mathrm{dm}^{-3}$; macroporos $=0,11 \mathrm{dm}^{3} \mathrm{dm}^{-3}$; e porosidade total = $0,52 \mathrm{dm}^{3} \mathrm{dm}^{-3}$. As densidades do solo e de partículas foram determinadas pelos métodos do anel e do balão volumétrico, respectivamente, e a granulometria, pelo método do densímetro, conforme Embrapa (1997). O $\mathrm{C}$ orgânico foi determinado por espectrofotometria; $\mathrm{P}$ foi extraído por solução Mehlich-1 e determinado por espectrofotometria; K foi extraído por solução Mehlich-1 e determinado por fotômetro de chama; $\mathrm{Ca}$ e $\mathrm{Mg}$ foram extraídos com $\mathrm{KCl} 1 \mathrm{~mol} \mathrm{~L}^{-1}$ e determinados por espectrofotometria de absorção atômica; $\mathrm{Al}$ foi extraído com $\mathrm{KCl} 1 \mathrm{~mol} \mathrm{~L}^{-1}$ e determinado por titulação $\mathrm{NaOH}$; o $\mathrm{pH}$ foi medido em solução de $\mathrm{CaCl}_{2}$ $10 \mathrm{mmol} \mathrm{L}^{-1}$, de acordo com Pavan et al. (1992); o ferro pedogenético ou ferro livre foi obtido por extrações com $\mathrm{DCB}$ e determinado por espectrofotometria de $\mathrm{ab}$ sorção atômica (Abreu Jr. et al., 2009); e a caulinita foi quantificada por termogravimetria, conforme Melo et al. (2002) (Quadro 1).

As colunas de solo foram coletadas em tubos de PVC, com altura de $25 \mathrm{~cm}$ e diâmetro interno de $20,3 \mathrm{~cm}$. Elas foram armazenadas em laboratório durante $54 \mathrm{~d}$ para perda de umidade e consequente contração do solo, o que permitiu criar um espaço entre a parede interna do tubo e a coluna de solo, de forma que possibilitasse vedar esse espaço, impedindo que a água de percolação formasse um fluxo preferencial através da parede do tubo.

As etapas que antecederam o processo de percolação foram: vedação, com silicone, do espaço entre a coluna de solo e a parede do tubo de PVC; colocação de uma camada de $0,5 \mathrm{~cm}$ de sílica com granulometria de $0,2 \mathrm{~cm}$, em pratos plásticos rígidos com $3,5 \mathrm{~cm}$ de altura; perfuração da parte central dos pratos para fixar uma mangueira com $1 \mathrm{~cm}$ de diâmetro, a fim de drenar o lixiviado; colocação dos pratos em uma bancada; colocação das colunas sobre os pratos; nivelamento das colunas; umedecimento do solo por meio da obstrução dos drenos com silicone; e manutenção durante $12 \mathrm{~h}$ de uma lâmina de água de $30 \mathrm{~mm}$. Esse procedimento foi repetido uma vez.

Foram aplicados os seguintes tratamentos em quatro repetições: (a) $\mathrm{AM}$ - adubo mineral com $\mathrm{KCl}$ solúvel, na quantidade de $0,615 \mathrm{~g} /$ coluna de $\mathrm{K}$ (100 kg ha-1), $\mathrm{P}$ solúvel $\mathrm{NH}_{4} \mathrm{H}_{2} \mathrm{PO}_{4}$ na quantidade de $0,25 \mathrm{~g} /$ coluna de $\mathrm{P}\left(78,6 \mathrm{~kg} \mathrm{ha}^{-1}\right)$ e $\mathrm{NH}_{4} \mathrm{NO}_{3}$ solúvel na quantidade de $0,45 \mathrm{~g} /$ coluna de $\mathrm{N}\left(140 \mathrm{~kg} \mathrm{ha}^{-1}\right)$; (b) AO - adubo orgânico com dejeto líquido de suíno, na quantidade de $485 \mathrm{~mL} /$ coluna $\left(150 \mathrm{~m}^{3} \mathrm{ha}^{-1}\right)$, equivalente a 0,63, 0,63 e 0,44 g/coluna de $\mathrm{K}$, Ca e $\mathrm{Mg}$, respectivamente, correspondendo a 195, $195 \mathrm{e}$ $127 \mathrm{~kg} \mathrm{ha}^{-1}$; (c) T-testemunha sem adubo.

Quadro 1. Teores de areia grossa (ag), areia fina (af), silte (s) e argila (a); concentrações de carbono orgânico (C), fósforo (P), potássio (K), cálcio (Ca), magnésio (Mg) e alumínio (Al); pH; e teor de caulinita (Ct) e de $\mathrm{Fe}_{2} \mathrm{O}_{3}$, nas camadas de solo de 0 a $2,5 \mathrm{~cm}$ e de 2,5 a $20 \mathrm{~cm}$

\begin{tabular}{|c|c|c|c|c|c|c|c|c|c|c|c|c|c|}
\hline \multirow{2}{*}{ Camada } & \multicolumn{13}{|c|}{ Características } \\
\hline & $\mathrm{Ag}$ & Af & $\mathbf{S}$ & A & $\mathrm{C}$ & $\mathbf{P}$ & $\mathbf{K}^{+}$ & $\mathrm{Ca}^{2+}$ & $\mathrm{Mg}^{2+}$ & $\mathrm{Al}^{3+}$ & pH & $\mathrm{Ct}$ & $\mathrm{Fe}_{2} \mathrm{O}_{3}$ \\
\hline $\mathrm{cm}$ & \multicolumn{5}{|c|}{$\mathrm{g} \mathrm{kg}^{-1}$} & $\mathrm{mg} \mathrm{kg}^{-1}$ & \multicolumn{4}{|c|}{$-\mathrm{mmol}_{\mathrm{c}} \mathrm{kg}^{-1}$} & & \multicolumn{2}{|c|}{ - dag $\mathrm{kg}^{-1}-$} \\
\hline $0-2,5$ & 39 & 59 & 219 & 683 & 21,4 & 65,3 & 8,15 & 68,3 & 36,8 & 0,0 & 6,3 & 68 & 21 \\
\hline $2,5-20$ & 38 & 43 & 184 & 735 & 11,9 & 52,5 & 2,75 & 41,5 & 20,5 & 0,0 & 5,3 & 58 & 22 \\
\hline
\end{tabular}


As adubações mineral (tratamento AM) e orgânica (tratamento $\mathrm{AO}$ ) corresponderam, aproximadamente, a três vezes as quantidades colocadas nas lavouras pelos agricultores - um critério adotado para simular o que ocorre quando a água de escoamento superficial, com alta carga de elementos químicos em solução, se infiltra na região do canal de um terraço.

No momento da coleta do dejeto de suíno foram retiradas quatro amostras desse material para determinar as concentrações de $\mathrm{K}, \mathrm{Ca}, \mathrm{Mg}$ e matéria seca (Brasil, 1982), cujas análises apresentaram, respectivamente, os teores de 1,3; 1,3; 0,9; e 63,3 $\mathrm{g} \mathrm{kg}^{-1}$. Também foi determinada a DQO do dejeto cujo valor médio foi de $20,1 \mathrm{~g} \mathrm{~L}^{-1} \mathrm{de}_{2}$.

Como a quantidade de dejeto líquido de suíno colocada em cada coluna de solo foi de $485 \mathrm{~mL}$ e apresentava baixos teores de matéria seca, o adubo mineral utilizado foi dissolvido em um volume de água deionizada para que em cada coluna fosse aplicada uma solução de $460 \mathrm{~mL}$. As colunas sem adubo (testemunha) receberam o mesmo volume de água. Esse procedimento foi executado de forma lenta, para que o solo absorvesse todo o volume da solução aplicada, e teve o objetivo de igualar a condição inicial de umidade de solo em cada coluna.

Imediatamente após a adição do adubo orgânico e mineral, foi aplicada água na superfície das colunas, por gotejamento, por meio de kits de soro hospitalar. Para melhor distribuição da água, foi colocado um feltro na superfície de cada coluna e três kits para cada coluna. Para evitar a formação de lâmina superficial de água, esta foi aplicada com uma vazão em torno de $2,2 \mathrm{~mL} \mathrm{~min}^{-1}$, por coluna.

A duração total do processo de percolação da água foi de 76 h e 30 min. A taxa de infiltração da solução nas colunas que receberam dejeto foi menor do que nos demais tratamentos, o que ocasionou diferença no tempo de coleta do lixiviado, tendo o dejeto suíno demandado tempo razoavelmente maior. No entanto, o volume de solução percolado em todos os tratamentos foi o correspondente a três volumes de poro, e o percolado foi coletado ininterruptamente em cinco turnos de mesmo volume. O volume de poros $\left(3.340 \mathrm{dm}^{3}\right)$ foi obtido a partir da porosidade total do solo $\left(0,52 \mathrm{dm}^{3} \mathrm{dm}^{-3}\right)$ e das dimensões da coluna (20,3 cm de diâmetro e $20 \mathrm{~cm}$ de altura). Assumiu-se que o efeito na retenção dos elementos minerais pela matriz do solo seria mais acentuado no primeiro volume de poros e, portanto, este foi subdividido em três turnos (T1A, T1B e T1C). Os demais turnos (T2 e T3) tiveram o volume de água coletada igual a um volume de poros. Assim, fez-se a coleta de cinco turnos, tendo os três primeiros o volume de 1,113 L cada, e os dois últimos, de 3,340 L cada. A água percolada foi coletada em um reservatório conectado ao dreno da coluna de solo, sendo retiradas duas amostras, uma de $200 \mathrm{~mL}$ e outra de $500 \mathrm{~mL}$, filtradas em filtro de $0,45 \mu \mathrm{m}$ e congeladas. Na amostra de $200 \mathrm{~mL}$ foram determinadas as concentrações de $\mathrm{K}, \mathrm{Ca}$ e Mg solúveis em espectrofotômetro de absorção atômica (PerkinElmer, 1973). A alíquota de $500 \mathrm{~mL}$ foi acidificada, para posterior análise da DQO.

Concluída a percolação da água, as colunas foram deixadas em repouso para eliminar a água residente na porosidade do solo. Após esse procedimento, as colunas de solo foram seccionadas em três camadas, 0 a $2,5 \mathrm{~cm}, 2,5$ a $10 \mathrm{~cm}$ e 10 a $20 \mathrm{~cm}$, para determinar o pH e os teores de $\mathrm{K}, \mathrm{Ca}, \mathrm{Mg}, \mathrm{Al}$ e matéria orgânica (Pavan et al., 1992).

Para a análise, foram utilizados dois esquemas fatoriais:

Fatorial 1 - em que foi utilizado o esquema fatorial $3 \times 5$, com quatro repetições, sendo os tratamentos constituídos por três adubações: adubo orgânico (AO), adubo mineral (AM) e testemunha (T); e cinco turnos: T1A, T1B, T1C, T2 e T3. As comparações múltiplas foram realizadas por contrastes ortogonais do teste $\mathrm{F}$ previamente estabelecidos: contraste 1 - T vs $\mathrm{AO}, \mathrm{AM}$; contraste 2 - $\mathrm{AO}$ vs AM, para os efeitos de tratamentos; e contraste 3 - T1A, T1B e T1C vs T2 e T3; contraste 4 - T1A vs T1B e T1C; contraste 5 - T1B vs T1C; e contraste 6 -T2 vs T3, para os efeitos de turnos.

Fatorial 2-em que foi utilizado o esquema fatorial $3 \times 3$, com quatro repetições, sendo os tratamentos constituídos por três adubações: adubo orgânico (AO), adubo mineral (AM) e testemunha (T); e três camadas de solo: de 0 a $2,5 \mathrm{~cm}$, de 2,5 a $10 \mathrm{~cm}$ e de 10 a $20 \mathrm{~cm}$. As comparações múltiplas foram realizadas por contrastes ortogonais do teste $\mathrm{F}$, previamente estabelecidos: contraste 1 - T vs AO, AM; e contraste 2 - AO vs AM, para os efeitos de tratamentos; e contraste $3-0$ a 2,$5 ; 2,5$ a 10 vs 10 a 20 ; e contraste $4-0$ a 2,5 vs 2,5 a 10 , para os efeitos de camadas de solo.

\section{RESULTADOS E DISCUSSÃO}

Os adubos influenciaram a lixiviação do K, uma vez que em todos os turnos as médias dos tratamentos $\mathrm{AO}$ e $\mathrm{AM}$ foram significativamente superiores às do tratamento $\mathrm{T}$ (Quadro 2). Os resultados devem-se à baixa afinidade do K com os constituintes do solo (Sparks, 1995). Na comparação entre adubos, as concentrações de K na água percolada não diferiram entre si, exceto no primeiro turno, em que o adubo mineral apresentou teor $21 \mathrm{mg} \mathrm{L}^{-1}$ mais elevado do que o do dejeto líquido de suíno, embora a quantidade de K aplicada via dejeto líquido de suíno (195 kg ha-1) tenha sido praticamente o dobro da aplicada via adubo mineral (100 kg ha-1). A menor lixiviação com a aplicação do dejeto deve-se possivelmente ao selamento superficial causado pela ação física das partículas sólidas do dejeto (Bertol et al., 2007), as quais ocuparam parte dos poros do solo, diminuindo assim a mobilidade do $\mathrm{K}$ em profundidade, conforme argumentado também por Peles (2007). 
Quadro 2. Valores médios e estimativas dos contrastes da concentração de $\mathrm{K}$ solúvel na água percolada nos turnos $T_{1 \mathrm{~A}}, \mathrm{~T}_{1 \mathrm{~B}}, \mathrm{~T}_{1 \mathrm{C}}, \mathrm{T}_{2}$ e $\mathrm{T}_{3}$, aplicados nos tratamentos adubo orgânico (AO), adubo mineral (AM) e testemunha $(\mathrm{T})$

\begin{tabular}{|c|c|c|c|c|c|}
\hline \multirow{2}{*}{ Turno } & \multicolumn{3}{|c|}{ Tratamento } & \multicolumn{2}{|c|}{ Estimativa do contraste } \\
\hline & AO & AM & $\mathbf{T}$ & T vs AO, AM & AO vs AM \\
\hline & \multicolumn{5}{|c|}{$m g L^{-1}$ de $K$} \\
\hline $\mathrm{T}_{1 \mathrm{~A}}(1,113 \mathrm{~L})$ & $14,0 \pm 0,6$ & $35,0 \pm 2,1$ & $14,0 \pm 0,9$ & $-21,0^{* * *}$ & $-21,0^{* * *}$ \\
\hline $\mathrm{T}_{1 \mathrm{~B}}(1,113 \mathrm{~L})$ & $24,8 \pm 1,2$ & $27,3 \pm 1,0$ & $14,3 \pm 1,3$ & $-23,5^{* * *}$ & $-2,5^{\mathrm{ns}}$ \\
\hline $\mathrm{T}_{1 \mathrm{C}}(1,113 \mathrm{~L})$ & $22,0 \pm 1,1$ & $25,0 \pm 1,2$ & $12,4 \pm 0,9$ & $-22,2^{* * *}$ & $-3,0 \mathrm{~ns}$ \\
\hline $\mathrm{T}_{2}(3,340 \mathrm{~L})$ & $20,1 \pm 2,0$ & $17,7 \pm 1,1$ & $6,6 \pm 0,3$ & $-24,6^{\text {***}}$ & $2,4 \mathrm{~ns}$ \\
\hline $\mathrm{T}_{3}(3,340 \mathrm{~L})$ & $17,3 \pm 1,2$ & $14,5 \pm 1,1$ & $8,2 \pm 0,5$ & $-15,4^{* * *}$ & $2,8 \mathrm{~ns}$ \\
\hline \multirow[t]{2}{*}{ Contraste } & \multicolumn{3}{|c|}{ Estimativa do contraste } & & \\
\hline & 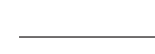 & $m g L^{-1}$ de $\mathrm{K}$ & 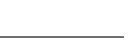 & & \\
\hline $\mathrm{T}_{1 \mathrm{~A}}, \mathrm{~T}_{1 \mathrm{~B}}, \mathrm{~T}_{1 \mathrm{C}}$ vs $\mathrm{T}_{2}, \mathrm{~T}_{3}$ & $9,4^{\mathrm{ns}}$ & $78,0^{* * *}$ & $37,0^{* * *}$ & & \\
\hline $\mathrm{T}_{1 \mathrm{~A}} \mathrm{vs} \mathrm{T}_{1 \mathrm{~B}}, \mathrm{~T}_{1 \mathrm{C}}$ & $-18,8^{* * *}$ & $17,7^{* * *}$ & $1,3^{\mathrm{ns}}$ & & \\
\hline $\mathrm{T}_{1 \mathrm{~B}}$ vs $\mathrm{T}_{1 \mathrm{C}}$ & $2,8^{\mathrm{ns}}$ & $2,3 \mathrm{~ns}$ & $1,9 \mathrm{~ns}$ & & \\
\hline $\mathrm{T}_{2} \mathrm{vs}_{3}$ & $2,8^{\mathrm{ns}}$ & $3,2^{\mathrm{ns}}$ & $-1,6^{\mathrm{ns}}$ & & \\
\hline
\end{tabular}

A concentração de K na água percolada diminuiu com o tempo de lixiviação, com maior intensidade no tratamento AM do que nos demais (Quadro 2). No tratamento $\mathrm{AO}$, a água percolada nos dois últimos volumes de poros $\left(\mathrm{T}_{2}\right.$ e $\left.\mathrm{T}_{3}\right)$ foi, respectivamente, $1 \mathrm{e}$ $15 \%$ menor do que a concentração média da água que percolou no primeiro volume de poros $\left(\mathrm{T}_{1 \mathrm{~A}}, \mathrm{~T}_{1 \mathrm{~B}} \mathrm{e}\right.$ $\mathrm{T}_{1 \mathrm{C}}$ ), enquanto no tratamento $\mathrm{AM}$ as reduções na concentração, nessa mesma ordem, foram em torno de 39 e $50 \%$.

O tipo de adubo também influenciou na lixiviação do Ca e do Mg. A concentração de Ca e Mg na água de percolação, na maioria dos turnos, foi maior no tratamento com adubo mineral do que no adubo orgânico, embora esses elementos tenham sido aplicados somente via dejeto líquido de suíno (Quadro 3). Esse comportamento pode ser explicado pela existência de $\mathrm{Ca}$ e $\mathrm{Mg}$ na estrutura orgânica do dejeto (Eck \& Stewart, 1995) e, portanto, não disponível para lixiviação a curto prazo, pela capacidade de compostos orgânicos em complexar íons como o Ca e o $\mathrm{Mg}$ (Sposito, 1989), bem como pelo impedimento físico exercido pelo material orgânico que permaneceu retido nos poros do solo (Oliveira et al., 2000; Bertol et al., 2007), conforme já mencionado para o $\mathrm{K}$, o qual possivelmente reduziu a mobilidade dos elementos solúveis no dejeto (Oliveira et al., 2000). A maior mobilidade do $\mathrm{Ca}$ e do $\mathrm{Mg}$ no tratamento $\mathrm{AM}$ provavelmente deveu-se ao K aplicado na forma de $\mathrm{KCl}$ nesse tratamento $\left(100 \mathrm{~kg} \mathrm{ha}^{-1}\right)$, tendo em vista que o K em elevadas concentrações tem a capacidade de aumentar temporariamente o deslocamento vertical do Ca e do $\mathrm{Mg}$, enquanto um novo equilíbrio entre esses cátions não for atingido (Sparks, 1995; Choudhary et al., 1996; Ernani et al., 2007). Quando um cátion qualquer é adicionado ao solo em grande quantidade, parte dos cátions preexistentes na camada elétrica difusa poderá ser lixiviada, uma vez que será deslocada para a solução do solo pelo cátion que entrou no sistema (Ernani, 2008). O aumento na lixiviação de $\mathrm{Ca}$ e $\mathrm{Mg}$ devido à aplicação de altas quantidades de $\mathrm{KCl}$ foi também constatado por Ernani et al. (2003).

As concentrações de $\mathrm{Ca}$ e $\mathrm{Mg}$ solúveis na água percolada diminuíram significativamente com o tempo de lixiviação, numa maior intensidade no tratamento $\mathrm{AM}$ do que nos demais, semelhante ao que ocorreu com o K. Os dados (Quadro 3) mostram que no tratamento $\mathrm{AO}$ as concentrações de $\mathrm{Ca}$ e $\mathrm{Mg}$ na água que percolou nos dois últimos volumes de poros $\left(\mathrm{T}_{2} \mathrm{e}\right.$ $\mathrm{T}_{3}$ ) foram, respectivamente, 36 e $12 \%$ menor do que a concentração média da água que percolou no primeiro volume de poros $\left(\mathrm{T}_{1 \mathrm{~A}}, \mathrm{~T}_{1 \mathrm{~B}}\right.$ e $\left.\mathrm{T}_{1 \mathrm{C}}\right)$, enquanto no tratamento AM as reduções na concentração, nessa mesma ordem, foram de 63 e $80 \%$. A redução na concentração de $\mathrm{Mg}$ na água que percolou nos dois últimos volumes de poro $\left(\mathrm{T}_{2} \mathrm{e}_{3}\right)$ no tratamento $\mathrm{AO}$ foi, respectivamente, de 60 e $26 \%$, em relação à concentração média da água que percolou no primeiro volume de poros $\left(\mathrm{T}_{1 \mathrm{~A}}, \mathrm{~T}_{1 \mathrm{~B}}\right.$ e $\left.\mathrm{T}_{1 \mathrm{C}}\right)$, ao passo que no tratamento AM as reduções, nessa mesma ordem, foram de 64 e $84 \%$. A semelhança nas reduções acentuadas verificadas na concentração de K (Quadro 2) e nas concentrações de Ca e Mg (Quadro 3) na água percolada nos turnos do tratamento $\mathrm{AM}$, em relação ao tratamento $\mathrm{AO}$, corrobora o argumento de que no tratamento $\mathrm{AO}$ parte da porosidade do solo foi ocupada pelo dejeto e, em razão disso, retardou a mobilidade também do Ca e do Mg neste tratamento, devido à redução da passagem da água de percolação (Peles, 2007; Bertol et al., 2007), associado à permanência do Ca e do $\mathrm{Mg}$ no solo, adsorvidos pela matéria orgânica do dejeto (Sposito, 1989), bem como 
Quadro 3. Valores médios e estimativas dos contrastes das concentrações de Ca e Mg solúveis na água percolada nos turnos $T_{1 \mathrm{~A}}, \mathrm{~T}_{1 \mathrm{~B}}, \mathrm{~T}_{1 \mathrm{C}}, \mathrm{T}_{2}$ e $\mathrm{T}_{3}$, aplicados nos tratamentos adubo orgânico (AO), adubo mineral (AM) e testemunha (T)



$\overline{* * * * * *}, \mathrm{e}^{\mathrm{ns}}$ : significativo a $0,1,1$ e $5 \%$ e não significativo a $5 \%$ pelo teste $\mathrm{F}$.

presente na estrutura orgânica do dejeto retida na porosidade. Os resultados fortalecem ainda o argumento de que o $\mathrm{K}$ aplicado em quantidade considerável no tratamento $\mathrm{AM}$, na forma de $\mathrm{KCl}$, teve a capacidade de aumentar a lixiviação do $\mathrm{Ca}$ e do $\mathrm{Mg}$ (Ernani, 2008). Os resultados mostram também que, com o avanço dos turnos, a concentração do $\mathrm{Mg}$ teve maior redução do que a do $\mathrm{Ca}$ em ambos os tratamentos, evidenciando maior mobilidade inicial do $\mathrm{Mg}$, o que é explicado pelo fato deesse elemento se ligar com menor energia às cargas negativas do solo do que o Ca (Sparks, 1995; Ernani, 2008).

A percentagem de $\mathrm{K}$ lixiviado, em relação ao aplicado no solo, foi acentuadamente maior no tratamento $\mathrm{AM}$ do que no $\mathrm{AO}$ em todos os turnos (Figura 1). Isso evidencia o já discutido: que o K contido no dejeto líquido de suíno foi menos propenso à lixiviação do que o contido no adubo mineral, concordando com Jakobsen (1996). Os resultados são ainda mais expressivos se considerado que a quantidade de $\mathrm{K}$ aplicada como adubo orgânico (195 kg ha-1) foi em torno de uma vez maior do que a quantidade aplicada como adubo mineral (100 kg ha-1).

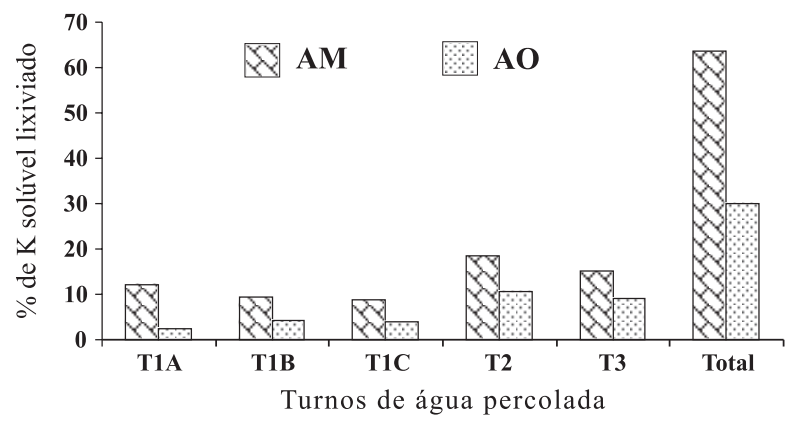

Figura 1. Percentagem (\%) de $\mathrm{K}$ solúvel lixiviado através das colunas de solo, nos turnos de água percolada $T_{1 \mathrm{~A}}, \mathrm{~T}_{1 \mathrm{~B}}, \mathrm{~T}_{1 \mathrm{C}}, \mathrm{T}_{2}$ e $\mathrm{T}_{3}$, em relação ao total de $\mathrm{K}$ aplicado nos tratamentos adubo mineral (AM) e adubo orgânico (AO).

A demanda química de oxigênio (DQO) da água percolada foi influenciada pelos adubos utilizados, uma vez que em todos os turnos a DQO nos tratamentos $\mathrm{AO}$ e AM foi superior à da testemunha (Quadro 4). O tipo de adubo também influenciou os resultados. A $\mathrm{DQO}$ da água que percolou no $\mathrm{AO}$ foi superior à do 
AM, exceto no primeiro turno, quando não houve diferença. Os resultados decorrem da contaminação da água pelos componentes orgânicos presentes no dejeto líquido de suíno.

$\mathrm{Na}$ comparação entre turnos, observa-se que a DQO do tratamento $\mathrm{AO}$ foi distinta em relação à do tratamento $\mathrm{AM}$; no tratamento $\mathrm{AO}$ os valores cresceram do primeiro para o segundo turno, diminuindo nos demais, porém de forma lenta. Já a $\mathrm{DQO}$ da água no tratamento AM reduziu progressivamente com o tempo de lixiviação. Os resultados demonstram que o dejeto líquido de suíno teve a capacidade de prolongar o seu efeito sobre a qualidade da água percolada. Isso torna-se mais evidente ao observar a DQO dos turnos finais $\left(\mathrm{T}_{2} \mathrm{e}\right.$ $\mathrm{T}_{3}$, os quais, conforme já mencionado, tiveram valores mais elevados no tratamento $\mathrm{AO}$ do que nos demais.

A comparação entre turnos evidencia ainda um efeito importante do $\mathrm{KCl}$ na movimentação de compostos orgânicos do solo no tratamento AM. Isso pode ser notado pela superioridade em todos os turnos da DQO da água que percolou através do solo que recebeu adubo mineral, em relação à DQO da água que percolou através do solo nativo (testemunha). Resultados semelhantes foram obtidos por Jones \& Willett (2006).

A mobilidade do K no solo foi influenciada pelo tipo de adubo (Quadro 5), uma vez que nas camadas de 0 a $2,5 \mathrm{~cm}$ e 2,5 a $10 \mathrm{~cm}$ a concentração desse elemento foi mais elevada no solo que recebeu dejeto líquido de suíno do que naquele que recebeu adubo mineral. Os resultados certamente devem-se ao efeito combinado da aplicação de uma quantidade mais elevada de $\mathrm{K}$ via dejeto líquido de suíno $\left(195 \mathrm{~kg} \mathrm{ha}^{-1}\right)$ do que via adubo mineral (100 $\left.\mathrm{kg} \mathrm{ha}^{-1}\right)$, com a menor lixiviação desse elemento no tratamento $\mathrm{AO}$ em relação ao tratamento AM (Quadro 2). Na comparação entre as camadas de solo, verifica-se que houve concentração maior desse elemento nas camadas de 0 a $2,5 \mathrm{~cm}$ e 2,5 a $10 \mathrm{~cm}$ do que na última camada (10 a $20 \mathrm{~cm}$ ). Esse efeito foi mais expressivo no tratamento $\mathrm{AO}$ do que no AM, provavelmente devido ao fato de que grande parte do dejeto permaneceu na porosidade das duas primeiras camadas de solo ( 0 a 2,5 cm e 2,5 a $10 \mathrm{~cm}$ ), associado ao fato de que a quantidade de $\mathrm{K}$ aplicada no tratamento $\mathrm{AO}$ foi praticamente o dobro da aplicada no tratamento AM.

O tipo de adubo exerceu influência na mobilidade do Ca e do Mg na primeira camada de solo. No solos com dejeto líquido de suíno, a concentração desses elementos foi superior à do solo com adubo mineral (Quadro 5). Os dados do solo estudado (Quadro 1) mostram $\mathrm{pH}$ em torno de 6 e uma mineralogia da fração argila dominada por caulinita, a qual se caracteriza por apresentar um ponto de carga zero a $\mathrm{pH}$ entre 3,5 e 4. Essa condição e os teores elevados de argila do solo possibilitaram uma CTC considerável (Fontes et al., 2001), que, associada à afinidade do Ca e Mg com os grupos fenólicos e carboxílicos dos coloides orgânicos no solo (Daniel et al., 1998; Souza et al., 2006), permitiu reter grande parte desses elementos na primeira camada do solo.

As camadas de solo do tratamento $\mathrm{AO}$ apresentaram concentrações de Ca e Mg significativamente diferentes entre si, tendo a camada superior (0 a $2,5 \mathrm{~cm})$ apresentado valores mais elevados (Quadro 5). Os resultados decorrem, certamente, do fato de o dejeto líquido de suíno ter sido aplicado em superfície sem incorporação no solo, associado à retenção em parte da porosidade da camada superior do solo, da porção

Quadro 4. Valores médios e estimativas dos contrastes da DQO da água percolada nos turnos $T_{1 \mathrm{~A}}, \mathrm{~T}_{1 \mathrm{~B}}, \mathrm{~T}_{1 \mathrm{C}}, \mathrm{T}_{2}$ e $\mathrm{T}_{3}$, aplicados nos tratamentos adubo orgânico (AO), adubo mineral (AM) e testemunha (T)

\begin{tabular}{|c|c|c|c|c|c|}
\hline \multirow{2}{*}{ Turno } & \multicolumn{3}{|c|}{ Tratamento } & \multicolumn{2}{|c|}{ Estimativa do contraste } \\
\hline & AO & AM & $\mathrm{T}$ & T vs AO, AM & AO vs AM \\
\hline & \multicolumn{5}{|c|}{$\longrightarrow$ DQO $\left(\mathrm{O}_{2} \mathrm{mg} \mathrm{L}^{-1}\right)$} \\
\hline $\mathrm{T}_{1 \mathrm{~A}}(1,113 \mathrm{~L})$ & $74,7 \pm 6,88$ & $95,5 \pm 10,8$ & $57,0 \pm 8,46$ & $-56,1^{*}$ & $-20,7^{\mathrm{ns}}$ \\
\hline $\mathrm{T}_{1 \mathrm{~B}}(1,113 \mathrm{~L})$ & $110,0 \pm 9,60$ & $84,0 \pm 9,54$ & $34,6 \pm 3,16$ & $-124,7^{* * *}$ & $25,9^{*}$ \\
\hline $\mathrm{T}_{1 \mathrm{C}}(1,113 \mathrm{~L})$ & $83,0 \pm 7,81$ & $49,3 \pm 9,51$ & $31,7 \pm 3,54$ & $-68,7^{* *}$ & $33,7^{* *}$ \\
\hline $\mathrm{T}_{2}(3,340 \mathrm{~L})$ & $75,2 \pm 8,76$ & $50,1 \pm 5,29$ & $37,4 \pm 4,57$ & $-50,6^{*}$ & $25,2^{*}$ \\
\hline $\mathrm{T}_{3}(3,340 \mathrm{~L})$ & $77,2 \pm 6,78$ & $40,1 \pm 3,34$ & $32,8 \pm 3,44$ & $-51,7^{* *}$ & $37,1^{* * *}$ \\
\hline \multirow[t]{2}{*}{ Contraste } & \multicolumn{3}{|c|}{ Estimativa do contraste } & & \\
\hline & $\longrightarrow$ & $\mathrm{DQO}\left(\mathrm{O}_{2} \mathrm{~L}^{-1}\right)$ & - & & \\
\hline $\mathrm{T}_{1 \mathrm{~A}}, \mathrm{~T}_{1 \mathrm{~B}}, \mathrm{~T}_{1 \mathrm{C}} \mathrm{vs}_{2}, \mathrm{~T}_{3}$ & $78,0^{\mathrm{ns}}$ & $187,1^{* * *}$ & $36,29^{\mathrm{ns}}$ & & \\
\hline $\mathrm{T}_{1 \mathrm{~A}}$ vs $\mathrm{T}_{1 \mathrm{~B}}, \mathrm{~T}_{1 \mathrm{C}}$ & $-43,4^{* *}$ & $57,7^{\mathrm{ns}}$ & $47,8^{* *}$ & & \\
\hline $\mathrm{T}_{1 \mathrm{~B}} \mathrm{vs}_{1 \mathrm{C}}$ & $26,9^{*}$ & $34,8^{* *}$ & $2,9^{\text {ns }}$ & & \\
\hline $\mathrm{T}_{2}$ vs $\mathrm{T}_{3}$ & $1,9^{\text {ns }}$ & $10,0^{\mathrm{ns}}$ & $4,6^{\mathrm{ns}}$ & & \\
\hline
\end{tabular}


Quadro 5. Valores médios e estimativas dos contrastes das concentrações de K, Ca e Mg nas camadas de solo de 0 a 2,5 cm, 2,5 a $10 \mathrm{~cm}$ e 10 a $20 \mathrm{~cm}$, após a percolação da água nos tratamentos adubo orgânico (AO), adubo mineral (AM) e testemunha (T)

\begin{tabular}{|c|c|c|c|c|c|}
\hline \multirow{2}{*}{ Camada de solo } & \multicolumn{3}{|c|}{ Tratamento } & \multicolumn{2}{|c|}{ Estimativa do contraste } \\
\hline & AO & AM & $\mathbf{T}$ & T vs AO, AM & AO vs AM \\
\hline $\mathrm{cm}$ & \multicolumn{5}{|c|}{$\mathrm{mmol}_{\mathrm{c}} \mathrm{dm}^{-3} \mathrm{de} \mathrm{K}$} \\
\hline 0 a 2,5 & $6,4 \pm 0,68$ & $4,7 \pm 0,26$ & $3,7 \pm 0,57$ & $-3,7^{*}$ & $1,7 \mathrm{~ns}$ \\
\hline 2,5 a 10 & $5,7 \pm 0,66$ & $3,7 \pm 0,29$ & $2,9 \pm 0,64$ & $-3,6^{*}$ & $2,1^{*}$ \\
\hline 10 a 20 & $2,3 \pm 0,10$ & $2,2 \pm 0,70$ & $1,4 \pm 0,31$ & $-1,7 \mathrm{~ns}$ & $0,1^{\mathrm{ns}}$ \\
\hline Contraste & \multicolumn{3}{|c|}{$\begin{array}{l}\text { Estimativa do contraste } \\
\mathrm{mmol}_{\mathrm{c}} \mathrm{dm}^{-3} \mathrm{de} \mathrm{K}\end{array}$} & & \\
\hline 0 a 2,$5 ; 2,5$ a 10 vs 10 a 20 & $7,5^{\text {***}}$ & $4,0^{* *}$ & $3,8^{*}$ & & \\
\hline \multirow[t]{2}{*}{0 a 2,5 vs 2,5 a 10} & $0,7^{\mathrm{ns}}$ & $1,1^{\mathrm{ns}}$ & $0,8^{\mathrm{ns}}$ & & \\
\hline & \multicolumn{3}{|c|}{$\mathrm{mmol}_{\mathrm{c}} \mathrm{dm}^{-3} \mathrm{de} \mathrm{Ca}$} & & \\
\hline 0 a 2,5 & $107,5 \pm 3,0$ & $47,8 \pm 5,6$ & $40,3 \pm 3,7$ & $-74,8^{* * *}$ & $59,8^{* * *}$ \\
\hline 2,5 a 10 & $40,0 \pm 6,0$ & $28,0 \pm 4,4$ & $30,5 \pm 6,1$ & $-7,0 \mathrm{~ns}$ & $12,0 \mathrm{~ns}$ \\
\hline 10 a 20 & $16,0 \pm 0,4$ & $15,5 \pm 3,0$ & $19,8 \pm 3,6$ & $8,0^{\mathrm{ns}}$ & $0,5^{\mathrm{ns}}$ \\
\hline Contraste & \multicolumn{3}{|c|}{$\begin{array}{l}\text { Estimativa do contraste } \\
\mathrm{mmol}_{\mathrm{c}} \mathrm{dm}^{-3} \mathrm{de} \mathrm{Ca}\end{array}$} & & \\
\hline 0 a 2,$5 ; 2,5$ a 10 vs 10 a 20 & $115,5^{* * *}$ & $44,8^{\text {** }}$ & 31,3 * & & \\
\hline \multirow[t]{2}{*}{0 a 2,5 vs 2,5 a 10} & $67,5^{* * *}$ & $19,8^{*}$ & $9,8^{\mathrm{ns}}$ & & \\
\hline & & $015+20$ & $\mathrm{~mol}_{\mathrm{c}} \mathrm{dm}^{-3}$ de $\mathrm{Mg}$ & & \\
\hline 0 a 2,5 & $86,8 \pm 2,3$ & $21,5 \pm 2,9$ & $\begin{array}{l}20,5 \pm 1,7 \\
14,3 \pm 3,3\end{array}$ & $\begin{array}{c}-67,3^{* * *} \\
-0.3 \mathrm{~ns}\end{array}$ & $\begin{array}{l}65,3^{* * *} \\
48^{\mathrm{ns}}\end{array}$ \\
\hline 10 a 20 & $\begin{array}{r}1,0 \pm 1,0 \\
8,0 \pm 0,4\end{array}$ & $7,3 \pm 0,85$ & $\begin{array}{r}14,0 \pm 0,0 \\
9,0 \pm 1,2\end{array}$ & 2,8 ns & $0,8^{\text {ns }}$ \\
\hline Contraste & \multicolumn{3}{|c|}{$\begin{array}{l}\text { Estimativa do contraste } \\
-\mathrm{mmol}_{\mathrm{c}} \mathrm{dm}^{-3} \mathrm{de} \mathrm{Mg}\end{array}$} & & \\
\hline 0 a 2,$5 ; 2,5$ a 10 vs 10 a 20 & $87,5^{* * *}$ & $19,0^{* *}$ & $16,8^{*}$ & & \\
\hline 0 a 2,5 vs 2,5 a 10 & $70,0^{* * *}$ & $9,5^{*}$ & $5,3^{\mathrm{ns}}$ & & \\
\hline
\end{tabular}

******* ens: significativo a $0,1,1$ e $5 \%$ e não significativo a $5 \%$ pelo teste $\mathrm{F}$.

sólida dos compostos orgânicos do dejeto, nos quais o $\mathrm{Ca}$ e $\mathrm{Mg}$ se encontravam particulados (Mendonça \& Rowell, 1994).

Comparando os resultados de concentração do K, $\mathrm{Ca}$ e $\mathrm{Mg}$ nas camadas de solo do tratamento $\mathrm{AO}$ (Quadro 5), observa-se que o primeiro teve maior mobilidade, uma vez que a concentração de $K$ na primeira camada ( 0 a $2,5 \mathrm{~cm}$ ) foi apenas $0,7 \mathrm{mmol}_{\mathrm{c}} \mathrm{dm}^{-3}$ mais elevada do que a da segunda $(2,5$ a $10 \mathrm{~cm})$, ao passo que para o $\mathrm{Ca}$ e o $\mathrm{Mg}$ as concentrações na primeira camada foram, respectivamente, 67,5 e $70,0 \mathrm{mmol}_{\mathrm{c}} \mathrm{dm}^{-3}$ superiores às da segunda. Os resultados, em parte, podem ser explicados considerando a série liotrófica dos principais cátions: $\mathrm{Ca}^{++}>\mathrm{Mg}^{++}>\mathrm{K}^{+}>\mathrm{NH}_{4}^{+}>\mathrm{Na}^{+}$(Sparks, 1995), a qual mostra que, em relação ao $\mathrm{Ca}$ e $\mathrm{Mg}$, o $\mathrm{K}$ tem menor reatividade com o solo.

O dejeto líquido de suíno alterou significativamente o $\mathrm{pH}$ da camada de solo de 0 a $2,5 \mathrm{~cm}$, elevando-o em uma unidade, contrariando a afirmativa de Scherer et al. (1996), de que é mínima a possibilidade de alteração no $\mathrm{pH}$ do solo por esse tipo de adubo. No entanto, resultados semelhantes foram obtidos por Hue (1992) e Iyamuremye et al. (1996), que os atribuíram ao aumento na solução do solo dos íons $\mathrm{OH}^{-}$, em decorrência das reações dos compostos orgânicos. Outros fatores podem igualmente ter contribuído para os resultados, como o aporte no solo de cátions básicos ( $\mathrm{Ca}, \mathrm{Mg}, \mathrm{K})$, a decomposição de ácidos graxos voláteis comuns nos resíduos fecais e o tamponamento dos dejetos suínos, que diminuem o impacto da acidificação via nitrificação (Sorensen, 1998).

$\mathrm{O}$ tipo de adubo influenciou a concentração de $\mathrm{Al}^{3+}$ na primeira camada de solo (0 a $2,5 \mathrm{~cm})$, uma vez que no tratamento $\mathrm{AO}$ essa camada não apresentou $\mathrm{Al}^{3+}$, enquanto no tratamento $\mathrm{T}$ a concentração foi de 2,8 mmolc dm $\mathrm{mm}^{-3}$ (Quadro 6). Os resultados, também constatados por Ceretta et al. (2003), devem-se, em grande parte, ao efeito do adubo orgânico, que elevou o pH do solo acima de 5,5, condição em que o $\mathrm{Al}$ trocável precipita-se como $\mathrm{Al}(\mathrm{OH})$ (Ernani, 2008) efeito similar ao proporcionado pelo calcário (Hue, 1992; Iyamuremye et al., 1996).

O teor de matéria orgânica no solo não foi alterado pelos adubos estudados, mesmo no tratamento AO, no qual os valores poderiam ser mais elevados, devido à adição do dejeto líquido de suíno, pelo menos na camada de 0 a $2,5 \mathrm{~cm}$ (Quadro 6). Os resultados podem 
Quadro 6. Valores médios e estimativas dos contrastes do $\mathrm{pH}$, da concentração de Al e do teor de matéria orgânica nas camadas de solo de 0 a 2,5, 2,5 a 10 e 10 a $20 \mathrm{~cm}$, após a percolação da água nos tratamentos adubo orgânico (AO), adubo mineral (AM) e testemunha (T)

\begin{tabular}{|c|c|c|c|c|c|}
\hline \multirow{2}{*}{ Camada de solo } & \multicolumn{3}{|c|}{ Tratamento } & \multicolumn{2}{|c|}{ Estimativa do contraste } \\
\hline & AO & $\mathbf{A M}$ & $\mathbf{T}$ & T vs AO, AM & AO vs AM \\
\hline $\mathrm{cm}$ & \multicolumn{5}{|c|}{$\mathrm{pH}\left(\mathrm{CaCl}_{2}\right)$} \\
\hline 0 a 2,5 & $5,7 \pm 0,00$ & $4,7 \pm 0,09$ & $4,8 \pm 0,1$ & $-0,8^{* * *}$ & $1,0^{* * *}$ \\
\hline 2,5 a 10 & $4,6 \pm 0,00$ & $4,6 \pm 0,07$ & $4,7 \pm 0,2$ & $0,1^{\mathrm{ns}}$ & 0,0 ns \\
\hline 10 a 20 & $4,5 \pm 0,04$ & $4,5 \pm 0,09$ & $4,5 \pm 0,1$ & $-0,1 \mathrm{~ns}$ & $0,1^{\text {ns }}$ \\
\hline Contraste & \multicolumn{3}{|c|}{$\begin{array}{r}\text { Estimativa do contraste } \\
\mathrm{pH}\left(\mathrm{CaCl}_{\diamond}\right)\end{array}$} & & \\
\hline 0 a 2,$5 ; 2,5$ a 10 vs 10 a 20 & $1,3^{* * *}$ & $0,4 \mathrm{~ns}$ & $0,6^{\mathrm{ns}}$ & & \\
\hline \multirow[t]{2}{*}{0 a 2,5 vs 2,5 a 10} & $1,1^{* * *}$ & $0,1 \mathrm{~ns}$ & $0,2^{\text {ns }}$ & & \\
\hline & \multicolumn{4}{|c|}{$\mathrm{mmol}_{\mathrm{c}} \mathrm{dm}^{-3} \mathrm{de} \mathrm{Al}^{3+}$} & \\
\hline 0 a 2,5 & 0,0 & $3,5 \pm 0,29$ & $2,8 \pm 0,25$ & $2,0^{* *}$ & $-3,5^{* * *}$ \\
\hline 2,5 a 10 & $5,0 \pm 0,41$ & $4,3 \pm 0,63$ & $4,8 \pm 0,63$ & $0,3 \mathrm{~ns}$ & $0,8^{\mathrm{ns}}$ \\
\hline 10 a 20 & $5,3 \pm 0,63$ & $5,8 \pm 0,95$ & $5,3 \pm 0,85$ & $-0,5 \mathrm{~ns}$ & $0,5^{\mathrm{ns}}$ \\
\hline Contraste & \multicolumn{3}{|c|}{$\begin{array}{l}\text { Estimativa do contraste } \\
\mathrm{mmol}_{\mathrm{c}} \mathrm{dm}^{-3} \mathrm{de} \mathrm{Al}^{3+}\end{array}$} & & \\
\hline 0 a 2,$5 ; 2,5$ a 10 vs 10 a 20 & $-5,5^{* * *}$ & $-3,8^{*}$ & $-3,0 \mathrm{~ns}$ & & \\
\hline \multirow[t]{2}{*}{0 a 2,5 vs 2,5 a 10} & $-5,0^{* * *}$ & $-0,8^{\mathrm{ns}}$ & $-2,0 \mathrm{~ns}$ & & \\
\hline & \multicolumn{4}{|c|}{ - $\mathrm{g} \mathrm{dm}^{-3}$ de matéria orgânica } & \\
\hline 0 a 2,5 & $39,3 \pm 3,8$ & $37,5 \pm 1,8$ & $39,0 \pm 2,0$ & $1,2 \mathrm{~ns}$ & $1,8^{\mathrm{ns}}$ \\
\hline 2,5 a 10 & $29,8 \pm 1,7$ & $28,5 \pm 1,9$ & $25,8 \pm 1,8$ & $-6,7 \mathrm{~ns}$ & $1,3 \mathrm{~ns}$ \\
\hline 10 a 20 & $29,0 \pm 5,1$ & $22,3 \pm 1,5$ & $21,0 \pm 1,8$ & $-9,3 \mathrm{~ns}$ & $6,8 \mathrm{~ns}$ \\
\hline Contraste & \multicolumn{3}{|c|}{$\begin{array}{l}\text { Estimativa do contraste } \\
\qquad \mathrm{g} \mathrm{dm}^{-3} \text { de matéria orgânica }\end{array}$} & & \\
\hline 0 a 2,$5 ; 2,5$ a 10 vs 10 a 20 & $11,1^{\mathrm{ns}}$ & $21,5^{* * *}$ & $22,8^{* * *}$ & & \\
\hline 0 a 2,5 vs 2,5 a 10 & $9,5^{\mathrm{ns}}$ & $9,0^{* *}$ & $13,2^{* * *}$ & & \\
\hline
\end{tabular}

ser creditados à baixa teor de matéria seca no dejeto $\left(63 \mathrm{~g} \mathrm{~kg}^{-1}\right)$, alterando pouco o teor de C no solo (Scherer et al., 1996). Outro fator que pode explicar os resultados é que possivelmente tenha ocorrido um processo de translocação dos compostos orgânicos de baixa massa molecular presentes no dejeto suíno, fazendo com que os teores de matéria orgânica se elevassem em todas as camadas, de forma a não haver entre elas diferença estatística. Isso pode ser observado pela análise de contraste apresentada no quadro 6. Somente o tratamento $\mathrm{AO}$ não mostrou diferença significativa no teor de matéria orgânica medido nas três camadas de solo. A existência de compostos orgânicos de baixo peso molecular em dejetos de suínos foi constatada por Brunetti et al. (2007). A possibilidade de ter ocorrido a translocação também é corroborada pelos resultados da DQO do tratamento AO (Tabela 4), os quais indicam, pelos valores elevados em todos os turnos de coleta de água percolada, que houve lixiviação de compostos orgânicos.

Tendo em vista que os resultados do presente estudo correspondem ao efeito de uma camada de solo de apenas $20 \mathrm{~cm}$, e considerando que pesquisas em sistemas de semeadura direta têm constatado elevada concentração de elementos solúveis no escoamento superficial (Allen \& Mallarino, 2008; Bertol et al., 2010), pode-se inferir que, numa condição de solos argilosos, altamente intemperizados e profundos, é recomendável a adoção de práticas que contenham o escoamento superficial e favoreçam a infiltração da água. Entre as práticas que contribuem para a infiltração da água no solo, o terraceamento bem dimensionado, por exemplo, constitui-se em uma alternativa adequada para tal, já que a água do escoamento será contida na região do canal do terraço e tenderá a se infiltrar no solo. Com isso, haverá redução da carga iônica presente no escoamento, com repercussões positivas nos aspectos econômico e ambiental, uma vez que, assim se fazendo, será evitada tanto a perda de nutrientes das lavouras quanto a contaminação das águas superficiais e subterrâneas (Bertol et al., 2007; Brock et al., 2007).

\section{CONCLUSÕES}

1. A lixiviação de $\mathrm{K}, \mathrm{Ca}$ e $\mathrm{Mg}$ foi maior no tratamento com adubo mineral comparado ao adubo orgânico, mesmo tendo sido aplicado menos $\mathrm{K}$ via adubo mineral. 
2. O dejeto líquido de suíno promoveu aumento do $\mathrm{pH}$ em uma unidade na camada mais superficial do solo (0 a $2,5 \mathrm{~cm})$, neutralizando o $\mathrm{Al}^{3+}$.

3. Os resultados de lixiviação e de retenção dos elementos químicos estudados demonstram a capacidade do Latossolo Vermelho eutroférrico típico em exercer a função de filtro, atenuando parcialmente a carga poluente de dejetos líquidos de suíno aplicados de forma superficial.

\section{LITERATURA CITADA}

ABREU JR., C.H.; MARTIN-NETO, L.; MILORI, D.M.B.P.; SIMOES, M.L. \& DA SILVA, W.T.L. Métodos analíticos utilizados em química de solo. In: ALEONI, L.R.F. \& MELO, V.F. Química e mineralogia do solo. Viçosa, MG, Sociedade Brasileira de Ciência do Solo, 2009. p.529-694.

ALLEN, B.L. \& MALLARINO, A.R. Phosphorus loss by surface runoff in no-till system under mineral and organic fertilization. J. Environ. Qual., 37:125-137, 2008.

BERENGUER, P.; CELA, S.; SANTIVERI, F.; BOIXADER, J. \& LLOVERAS, J. Copper and zinc soil accumulation and plant concentration in irrigated maize fertilized with liquid swin manure. Agron. J., 100:1056-1061, 2008.

BERTOL, O.J.; RIZZI, N.E.; BERTOL, I. \& ROLOFF, G. Perdas de solo e água e qualidade do escoamento superficial associadas à erosão entre sulcos em área cultivada sob semeadura direta e submetida às adubações mineral e orgânica. R. Bras. Ci. Solo, 31:781-792, 2007.

BERTOL, O.J.; RIZZI, N.E.; FAVARETTO, N. \& LANA, M.C. Effect of liquid swine manure rate, incorporation, and timing of rainfall on phosphorus loss with surface runoff. Sci. Agric., 67:71-77, 2010.

BERTONI, J. \& LOMBARDI NETO, F. Conservação do solo. São Paulo, Ícone, 1990. 355p.

BRASIL. Ministério da Agricultura. Métodos padrões oficiais para a análise de fertilizantes. Portaria SNAD n. 31, Laboratório de Referência Vegetal. Brasília, 08 de jun. 1982.

BROCK, E.H.; KETTERINGS, Q.M. \& KLEINMAN, P.J.A. Phosphorus leaching through intact soil cores as onfluenced by type and duration of manure application. Nutr. Cycl. Agroecosyst., 77:269-281, 2007.

BRUNETTI, G.; PLAZA, C.; CLAPP, C.E. \& SENESI, N. Compositional and functional features of humic acids from organic amendments and amended soils in Minnesota, USA . Soil Biol. Biochem., 39:1355-1365, 2007.

CERETTA, C.A.; DURIGON, R.; BASSO, C.J.; BARCELLOS, L.A.R. \& VIEIRA, F.C.B. Características químicas de solo sob aplicação de esterco líquido de suínos em pastagem natural. Pesq. Agropec. Bras., 38:729-735, 2003.

CHOUDHARY, L.D.; BAILEY, L.D. \& GRANT, C.A. Review of the use of swine manure in crop production: Effects on yield and composition and on soil and water quality. Waste Manag. Res., 14:581-595, 1996.
CONTE, E.; ANGHINONI, I. \& RHEINHEIMER, D.S. Frações de fósforo acumuladas em Latossolo argiloso pela aplicação de fosfato no sistema plantio direto. R. Bras. Ci. Solo, 27:893-900, 2003.

DANIEL, T.C.; SHARPLEY, A.N. \& LEMUNYON, J.L. Agricultural phosphorus and eutrofication: A symposium overview. J. Environ. Qual., 27:251-257, 1998.

ECK, H.V. \& STEWART, B.A. Manure. In: RECHCIGL, J.E., ed. Soil amendments and environmental quality. New York, 1995. p.169-198.

EMPRESA BRASILEIRA DE PESQUISA AGROPECUÁRIA EMBRAPA. Centro Nacional de Pesquisa de Solos. Manual de métodos de análise de solo. 2.ed. Rio de Janeiro, 1997. $212 \mathrm{p}$

ERNANI, P.R.; MANTOVANI, A.; SCHEIDT, F.R. \& NESI, C. Mobilidade de nutrientes em solos ácidos decorrentes da aplicação de cloreto de potássio e calcário. In: CONGRESSO BRASILEIRO DE CIÊNCIA DO SOLO, 29., Ribeirão Preto, 2003. Resumos expandidos. Ribeirão Preto, Sociedade Brasileira de Ciência do Solo, 2003. CD ROM.

ERNANI, P.R.; BAYER, C.; ALMEIDA, J.A. \& CASSOL, P.C. Mobilidade vertical de cátions influenciada pelo método de aplicação de cloreto de potássio em solos com carga variável. R. Bras. Ci. Solo, 31:393-402, 2007.

ERNANI, P.R. Química do solo e disponibilidade de nutrientes. Lages, Evangraf, 2008. 230p.

FONTES, M.P.F.; CAMARGO, O.A. \& SPOSITO, G. Eletroquímica das partículas coloidais e sua relação com a mineralogia de solos altamente intemperizados. Sci. Agric., 58:627-646, 2001.

GOMES, P.C.; FONTES, M.P.F.; SILVA, A.G.; MENDONÇA, E.S.; ANDRÉ R. \& NETTO, A.R. Selectivity sequence and competitive adsorption of heavy metals by brazilian soils. Soil. Soil Sci. Soc. of Am. J., 65:1115-1121, 2001.

GRIEBELER, N.P.; PRUSKI, F.F.; TEIXEIRA, A.F. \& SILVA, D.D. Modelo para o dimensionamento e a locação de sistemas de terraceamento em nível. Eng. Agríc., 25:696704, 2005.

HUE, N.V. Correcting soil acidity of a highly weathered ultisol with chiken manure and sewage sludge. Comm. Soil Sci. Plant Anal., 23:242-264, 1992.

IYAMUREMYE, F.; DICK, R.P \& BAHAM, J. Organic amendments and phosphorus dynamics: i. phosphorus chemistry and sorption. Soil Sci., 161:426-435, 1996.

JAKOBSEN, S.T. Leaching of nutrients from pots with and without apllied compost. Res. Conserv. Recycl., 1996. p.1-11.

JONES, D.L. \& WILLETT, V.B. Experimental evaluation of methods to quantify dissolved organic nitrogen (DON) and dissolved organic carbon (DOC) in soil. Soil Biol. Biochem., 38:991-999, 2006.

LARSON, W.E. \& PIERCE, F.J. Conservation and enhancement of soil quality. In: INTERNATIONAL BOARD FOR SOIL RESEARCH AND MANAGEMENT, Bangkok, 1991. Proceedings... Bangkok, ISBRAM, 1991. v.2. p.175-203. 
McDOWELL, R. \& SHARPLEY, A.N. Phosphorus losses in subsurface flow before and after manure application to intensively farmed land. Sci. Total Environ., 278:113-125, 2001.

MELO, V.F.; SCHAEFER, M.P.F.; NOVAIS, R.F.; SINGH, B. \& FONTES, M.P.F. Potassium and magnesium in clay minerals of some Brazilian soils as incated by a sequential extraction procedure. Comm. Soil Sci. Plant Anal., 33:22032225,2002

MENDONÇA, E.S. \& ROWEL, D.L. Dinâmica do alumínio e de diferentes frações orgânicas de um Latossolo argiloso sob cerrado e soja. R. Bras. Ci. Solo, 18:295-303, 1994.

OLIVEIRA, R.A.; CAMPELO, P.L.G.; MATOS, A.T.; MARTINEZ, M.A. \& CECON, P.R. Influência da aplicação de águas residuárias de suinocultura na capacidade de infiltração de um solo Podzólico Vermelhoamarelo. R. Bras. Eng. Agric. Amb., 4:263-267, 2000.

PARHAM, J.A.; DENG. S.P.; RAUN, W.R. \& JOHNSON, G.V. Long-term cattle manure application In: Effect on soil phosphorus levels, microbial biomass C, and dehydrogenese and phosphatase activities. Biol. Fert. Soil, 35:328-337, 2002.

PAVAN, M.A.; BLOCH, M.F.; ZEMPULSKI, H.C.; MIYAZAWA, M. \& ZOCOLER, D.C. Manual de analise química de solo e controle de qualidade. Londrina, Instituto Agronômico do Paraná, 1992. 38p. (Circular, 76)

PELES, D. Perdas de solo, água e nutrientes sob aplicação de gesso e dejeto líquido de suínos. Curitiba, Universidade Federal do Paraná, 2007. 84p. (Tese de Mestrado)

PERKIN-ELMER, N. Analytical methods for atomic absorption spectrophotometry agriculture. Connecticut, PerkinElmer, 1973.

PIOVESAN, R.P.; FAVARETTO, N.; PAULETTI, V.; MOTTA, A.C.V. \& REISSMANN, C.B. Perdas de nutrientes via subsuperfície em colunas de solo sob fertilização mineral e orgânica. R. Bras. Ci. Solo, 33:757-766, 2009.
PREVEDELLO, C.L. Física do solo. Curitiba, SAEFS, 1996. $446 \mathrm{p}$.

SCHERER, E.E.; AITA, C. \& BALDISSERA, I.T. Avaliação da qualidade do esterco líquido de suíno da região oeste catarinense para fins de utilização como fertilizante. Florianópolis, EPAGRI, 1996. 46p. (Boletim Técnico, 79)

SHIGAKI, F.; SHARPLEY, A. \& PROCHNOW, L.I. Sourcerelated transport of phosphorous in sourface runoff. J. Environ. Qual., 35:2229-2235, 2006.

SIMARD, R.R.; BEAUCHEMIN, S. \& HAYGARTH, P.M. Potential for preferential pathways of phosphorus transport. J. Environ. Qual., 29:97-105, 2000.

SIMS, J.T.; SIMARD, R.R. \& JOERN, B.C. Phosphorus loss in agricultural drainage: Historical perspective and current research. J. Environ. Qual., 27:277-293, 1998.

SØRENSEN, P. Carbon mineralization, nitrogen immobilization and $\mathrm{pH}$ change in soil after adding volatile fatty acids. Europ. J. Soil Sci., 49:457-462, 1998.

SPARKS, D.L. Environmental soil chemistry. San Diego, Academic Press, 1995. 267p.

SPOSITO, G. The chemistry of soils. New York, Oxford University Press, 1989. 277p.

SOUZA, F.R.; FAQUI, V.; TORRES, P.R.F. \& BALIZA, D.P. Calagem e adubação orgânica: Influência na adsorção de fósforo em solo. R. Bras. Ci. Solo, 30:975-983, 2006.

TAKALSON, D.D. \& LEYTEM, A.B. Phosphorus mobility in soil columns treated with dairy manures and commercial fertilizer. Soil Sci., 174:73-80, 2009. 
